\title{
Searching Less Perturbed Circular Orbits for a Spacecraft Travelling around Europa
}

\author{
J. P. S. Carvalho, ${ }^{1}$ R. Vilhena de Moraes, ${ }^{1}$ and A. F. B. A. Prado ${ }^{2}$ \\ ${ }^{1}$ Instituto de Ciência e Tecnologia, Universidade Federal de São Paulo (UNIFESP), Rua Talim 330, \\ 12231-280 São José dos Campos, SP, Brazil \\ ${ }^{2}$ Instituto Nacional de Pesquisas Espaciais, Avenida dos Astronautas 1758, 12243-750 São José dos Campos, SP, Brazil
}

Correspondence should be addressed to J. P. S. Carvalho; jeanfeg@gmail.com

Received 31 March 2014; Revised 23 May 2014; Accepted 6 June 2014; Published 26 June 2014

Academic Editor: Vivian Martins Gomes

Copyright (C) 2014 J. P. S. Carvalho et al. This is an open access article distributed under the Creative Commons Attribution License, which permits unrestricted use, distribution, and reproduction in any medium, provided the original work is properly cited.

\begin{abstract}
Space missions to visit the natural satellite of Jupiter, Europa, constitute an important topic in space activities today, because missions to this moon are under study now. Several considerations have to be made for these missions. The present paper searches for less perturbed circular orbits around Europa. This search is made based on the total effects of the perturbing forces over the time, evaluated by the integral of those forces over the time. This value depends on the dynamical model and on the orbit of the spacecraft. The perturbing forces considered are the third-body perturbation that comes from Jupiter and the $J_{2}, J_{3}$, and $C_{22}$ terms of the gravitational potential of Europa. Several numerical studies are performed and the results show the locations of the less perturbed orbits. Using those results, it is possible to find near-circular frozen orbits with smaller amplitudes of variations of the orbital elements.
\end{abstract}

\section{Introduction}

The importance of making a good choice for the orbit of a spacecraft that will make a mission around Europa can be explained in several aspects. First, this choice will impact on the scientific results that can be obtained from the observation points that the chosen orbit allows. Second, it is necessary to consider the orbital maneuvers involved in placing and keeping the spacecraft on that orbit. This problem impacts on the costs and duration of the mission, so it is an aspect that has to be considered during the orbit selection process. Some researches on this topic are described below.

Reference [1] studied the stability of orbits around a planetary satellite using analytical and numerical techniques with applications in Europa. The solution includes the effects of the planet gravity and the planetary satellite oblateness, with the assumption that the eccentricity of the nominal orbit is small. After that [2] studied the secular motion of a spacecraft around Europa that is in a synchronous orbit. Lara and SanJuan [3] investigated the stability of the motion in the threedimensional space for the Jupiter-Europa system. Lara and
Russell [4] considered the dynamics of a satellite around Europa taking into account the model of Hill and the Europa gravity field $\left(\mathrm{J}_{2}\right.$ and $\left.\mathrm{J}_{3}\right)$ on the synchronous moon theory. Paskowitz and Scheeres [5] analyzed the dynamics of an orbit around Europa taking into account a model that includes the tidal force of Jupiter and $J_{2}, C_{22}$, and $J_{3}$ gravity coefficients of Europa. Average techniques are applied twice to reduce the original 3-degree-of-freedom system to an integrable 1degree-of-freedom system. After that $[6,7]$ obtained orbits for a scientific mission in Europa, while [8] studied how to control this type of orbits. Russell and Brinckerhoff [9] studied eccentric orbits around several moons of the solar system. Lara [10] reviewed the long-term dynamics of an orbiter around a planetary moon. Application to the Jupiter-Europa system illustrates the full procedure. More recently, in Carvalho et al. [11], the dynamics of orbits around planetary satellites, taking into account the gravitational attraction of a third body and the nonuniform distribution of mass of the planetary satellite, is studied. The authors show that the singleaveraged model is more realistic, since it does not eliminate the term due to the equatorial ellipticity of the planetary 
satellite as done by the double-averaged problem. Just after that, Carvalho et al. [12] analyzed the problem of considering that an artificial satellite is orbiting Europa and that this spacecraft is perturbed by the nonuniform distribution of mass of the planetary satellite $\left(J_{2}, J_{3}\right.$, and $\left.C_{22}\right)$ and by the gravitational attraction of the third body. In this paper, the authors present an analytical theory using the averaged model and numerical simulations are performed using the software Mercury ([13]), to compare the results obtained using the analytical theory. In the following year, Carvalho et al. [14] search for orbits around Europa with longer lifetimes. The dynamical model considers the effects caused by the nonsphericity $\left(J_{2}, J_{3}\right)$ of the central body (Europa) and the perturbations caused by a third body in the motion of an artificial satellite around Europa. The orbit of Jupiter is assumed to be circular and fixed in the space; therefore, the mean anomaly of Jupiter has not been eliminated.

The goal of the present paper is to map orbits around Europa by performing the integral over the time of the most important perturbations, in order to evaluate the total effects of those forces involved in the dynamics. This search for orbits that are less perturbed is based on the fact that it is expected that less perturbed orbits suffer smaller deviations from the nominal orbits and therefore demand less stationkeeping maneuvers. This idea is first used and more explained in Prado [15], who studied spacecraft orbits around the Earth perturbed by the moon and the sun using this technique. To make a first study for orbits around Europa using this concept, only circular orbits are considered, but the semimajor axis and the inclinations are free to assume any desired value. In this way, the results will allow mission designers to choose the best orbits that are suitable for their missions with respect to this aspect. This criterion for mapping the orbits has several advantages. Some of them are as follows.

(1) The orbits are assumed to be always keplerian, due to the fact that the integration is performed for only one period of the spacecraft that is a short time. In this way, this index can be evaluated for each perturbation force independent from the others. So, it is possible to make a comparison of those values to choose which forces are important and need to be considered in the dynamical model, depending on the accuracy desired by the mission.

(2) This index depends on the force model used and the orbit of the spacecraft. It does not depend on the propulsion system and control techniques used for the station keeping.

(3) It measures the total amount of variation of the velocity that comes from the perturbation forces considered in the dynamical model; therefore, it indicates which orbits are good candidates to require less fuel consumption for the station-keeping maneuvers.

After mapping orbits with respect to the perturbations, the results are used to find the values for the semimajor axis that generate less perturbed frozen orbits. A frozen orbit is a specific orbit where the perturbations are minimized by making a good choice of the orbital parameters of that orbit.
TABle 1: Numerical values for $J_{2}, J_{3}$, and $C_{22}$.

\begin{tabular}{l}
\hline Harmonic coefficients of Europa (Lara and Russell [4]) \\
\hline$J_{2}=4.35 \times 10^{-4}$ \\
$J_{3}=1.378 \times 10^{-4}$ \\
$C_{22}=1.307 \times 10^{-4}$
\end{tabular}

Therefore, the use of values for the semimajor axis based on this study can lead to the discovery of frozen orbits with smaller variations of the orbital parameters that can be used in several missions.

\section{Mathematical Models}

The mathematical model used here for the perturbations is now explained in some detail. The problem deals with a system of nonlinear differential equations, and we are using an extension of the theory proposed by Prado [15] to map a class of particular solutions. It is assumed that there is a spacecraft in a circular orbit around Europa. The forces acting in this spacecraft are the gravitational field of Europa, considering the terms $J_{2}, J_{3}$, and $C_{22}$, and the third-body perturbation due to Jupiter. Those terms were chosen because they are the most important ones in the gravitational potential of Europa. Table 1 shows the numerical values of those constants. Note that the values are of the same order of magnitude; therefore, it is necessary to take all of them into account in the dynamical model. Other perturbations, like the gravitational field of the other moons of Jupiter, are relevant only for very specific orbits, near the orbits of one of those moons, so they are not included in the present research. The equations that describe the force field are also shown in Table 1.

2.1. Force Function due to the Disturbing Body. For the model considered in the present paper, it is necessary to calculate the term $R_{2}$ of the disturbing function due to the perturbation caused by a third body (Jupiter) in circular orbits around Europa. This disturbing potential $R_{2}$ can be written in the form $([11,12,16])$

$$
R_{2}=\frac{1}{2} N^{2} r^{2}\left(3 \cos ^{2} S-1\right)
$$

where $r$ is the radius vector of the artificial satellite, $N$ is the mean motion of Jupiter, and $S$ is the angle between the line that connects the massive central body and the perturbed body (the artificial satellite) and the line that connects the massive central body and the perturbing body (Jupiter, the third body). The artificial satellite is considered to be a point of mass in a circular orbit with osculating orbital elements given by $a=$ (semimajor axis), $i$ (inclination), $g$ (argument of the periapsis), and $h$ (longitude of the ascending node). Using the relation between the angle $S$ and the true anomaly $(f)$ of the satellite, it is possible to find [17]

$$
\cos (S)=\alpha \cos (f)+\beta \sin (f) .
$$


For the case of circular orbits, the values of $\alpha$ and $\beta$ can be written in the form $[17,18]$

$$
\begin{gathered}
\alpha=\cos (g) \cos (h-M)-\cos (i) \sin (g) \sin (h-M), \\
\beta=-\sin (g) \cos (h-M)-\cos (i) \cos (g) \sin (h-M),
\end{gathered}
$$

where $M$ is the true anomaly of Jupiter. For the case of elliptical orbits, $\alpha$ and $\beta$ are presented in [19], where it is considered that the satellite is in the same plane of the disturbing body. However, when the inclination of the orbit of the disturbing body (in an elliptical orbit) is taken into account, $\alpha$ and $\beta$ are given in [20]. Then, (2) and (3) are used in (1) and the disturbing potential can be written as

$$
\begin{aligned}
R_{2}=- & \frac{3}{8} r^{2} N^{2} \\
\times( & -\frac{1}{2}(c-1)^{2} \cos (2 g-2 h+2 M+2 f) \\
& -\frac{1}{2}(c+1)^{2} \cos (2 g+2 h-2 M+2 f) \\
& -\cos (2 h-2 M)-\cos (2 g+2 f) \\
& \left.+\frac{1}{3}+c^{2} \cos (2 g+2 f)-c^{2}+c^{2} \cos (2 h-2 M)\right) .
\end{aligned}
$$

We use the shortcuts $s=\sin (i)$ and $c=\cos (i)$.

The potential of a massive body at a distance $r$ and the force per unit of mass are related by (McCuskey) [21]

$$
F=-\frac{\partial U}{\partial r}
$$

where $U$ is the potential to be considered.

Replacing (4) in (5) and using $r=a, f=n t$, and $M=N t$, where $n$ is the mean motion of the satellite, $N$ is the mean motion of Jupiter, and $t$ is the time, it is obtained that

$$
\begin{aligned}
F R_{2}=\frac{3}{4} a N^{2} & \\
\times( & -\frac{1}{2}(c+1)^{2} \cos (2 g+2 h-2 N t+2 n t) \\
& -\frac{1}{2}(c-1)^{2} \cos (2 g-2 h+2 N t+2 n t) \\
& +\frac{1}{3}-\cos (2 N t-2 h)-c^{2}+c^{2} \cos (2 N t-2 h) \\
& \left.-\cos (2 n t+2 g)+c^{2} \cos (2 n t+2 g)\right) .
\end{aligned}
$$

2.2. Force Function due to the Nonspherical Shape of Europa. To analyze the motion of a spacecraft around the planetary satellite, it is necessary to take into account the nonsphericity of Europa. As mentioned above, the harmonic coefficients $J_{2}$, $J_{3}$, and $C_{22}$ are only considered, as usual in the literature. Then, considering the case of circular orbits, the following equations are available. (a) The zonal perturbation due to the oblateness $\left(J_{2}\right)$ is given by [22]

$$
F J_{2}=-\frac{\varepsilon \mu_{E}\left(3 s^{2} \sin ^{2}(f+g)-1\right)}{2 r^{3}},
$$

where $\varepsilon=J_{2} R_{E}^{2}, R_{E}$ is the equatorial radius of Europa $\left(R_{E}=1560.8 \mathrm{~km}\right)$, and $\mu_{E}$ is the gravitational constant of Europa. Replacing (7) in (5) and performing some algebraic manipulations, it is possible to find the result

$\mathrm{FJ}_{2}$

$=-\frac{3 \varepsilon n^{2}\left(1-3 c^{2}-3 \cos (2 n t+2 g)+3 c^{2} \cos (2 n t+2 g)\right)}{2 a}$.

(b) The zonal perturbation due to the pear-shaped $\left(J_{3}\right)$ is defined by [21]

$F J_{3}=-\frac{\varepsilon_{1} \mu_{E}\left(5 s^{3} \sin ^{3}(f+g)-3 s \sin (f+g)\right)}{2 r^{4}}$,

where $\varepsilon_{1}=J_{3} R_{E}^{3}$. Replacing (9) in (5), after some algebraic manipulations, the result is

$$
\begin{aligned}
F J_{3}= & -\left(\varepsilon _ { 1 } n ^ { 2 } s \left(15 s^{2} \sin (n t+g)\right.\right. \\
& \left.\left.-5 s^{2} \sin (3 n t+3 g)-12 \sin (n t+g)\right)\right) \\
& \times\left(2 a^{2}\right)^{-1} .
\end{aligned}
$$

(c) For the sectorial perturbation $\left(J_{22}\right)$, the resultant equation is [22]

$$
\begin{aligned}
F C_{22}= & \left(\delta \mu _ { E } \left(6 \xi^{2} \cos ^{2}(f)+6 \chi^{2} \sin ^{2}(f)+6 \xi \chi \sin (2 f)\right.\right. \\
& \left.\left.-3+3 s^{2} \sin ^{2}(f+g)\right)\right) \\
& \times\left(r^{3}\right)^{-1},
\end{aligned}
$$

where $\delta=C_{22} R_{E}^{2}$ and

$$
\begin{gathered}
\xi=\cos (g) \cos (h)-c \operatorname{sen}(g) \operatorname{sen}(h), \\
\chi=-\operatorname{sen}(g) \cos (h)-c \cos (g) \operatorname{sen}(h) .
\end{gathered}
$$

Replacing (11) in (5) and making some algebraic manipulations,

$$
\begin{aligned}
F C_{22}=\left(9 n ^ { 2 } \delta \left((c-1)^{2} \cos (2 n t+2 g-2 h)\right.\right. \\
+(c+1)^{2} \cos (2 n t+2 g+2 h) \\
\left.\left.+2 \cos (2 h)-2 c^{2} \cos (2 h)\right)\right)
\end{aligned}
$$


2.3. Effects of the Forces Involved in the Dynamics. The main effect of the forces involved in the dynamics during one period of the spacecraft given by (6), (8), (10), and (13) is to change the velocity $(V)$ of the satellite according to the physical law:

$$
\int_{0}^{T} F d t=\Delta V
$$

where $F$ is the force per unit of mass, $T$ is the period of the orbit of the spacecraft, and $F=F R_{2}+F J_{2}+F J_{3}+F C_{22}$. The integral given in (14) will be called PI (perturbation integral) due to its physical meaning and it will be measured in $\mathrm{km} / \mathrm{s}$. Therefore, according to Prado [15],

$$
\int_{0}^{T} F d t=\mathrm{PI}
$$

Equation (15) is numerically integrated to analyze the influence of each perturbation force in the orbit of an artificial satellite around Europa.

\section{Results}

The main goal of the present paper is to map orbits based on the evolution of the integral of the perturbing forces over the time for a spacecraft around Europa, as explained before.

\subsection{Studying the Effects of the Number of Revolutions. The} first step is to make a preliminary study to know how this parameter changes with the number of revolutions. This step is necessary in order to define the number of periods that gives a good view of the system, in terms of average. To accomplish this task, a circular orbit with semimajor axis of $1670 \mathrm{~km}$ and inclination of 90 degrees is used. The detailed results are not presented here, but they show that there is a repetition of values of the perturbation integral after $20 \mathrm{rev}-$ olutions; therefore, this number is assumed to represent very well the averaged system. Different values for the semimajor axis and inclination confirmed that the number of 20 revolutions is appropriated. So, from now on, all the values plotted for the perturbation integral will be obtained by integrating the perturbing forces for 20 revolutions of the spacecraft and then dividing it by 20 , to get an average value for each orbit. The numerical values for the physical parameters of Jupiter and Europa used in the present paper are $N=2.0477 \times$ $10^{-5} \mathrm{rad} / \mathrm{s}$ and $\mu_{E}=3202.7 \mathrm{~km}^{3} / \mathrm{s}^{2}$.

3.2. Mapping Orbits around Europa. After making this decision, Figure 1 is constructed considering the value for the perturbation integral as the average over 20 revolutions. It shows the value of the integral in the vertical axis (in $\mathrm{km} / \mathrm{s}$ ) and the inclination of the orbits (in degrees) in the horizontal axis. It is also important to take into account that since the integrals are taken over 20 revolutions of the spacecraft, the result is proportional to the period of the orbits. To be able to make comparisons among orbits that have different semimajor axes (therefore different periods, based on Kepler's Laws), it is necessary to choose one orbit to be the reference.

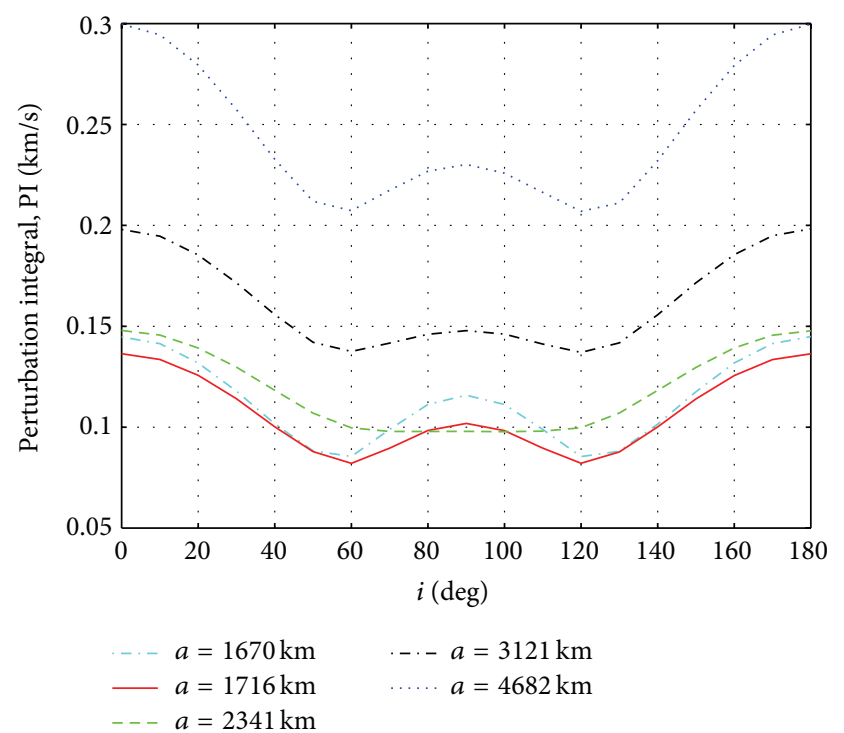

FIGURE 1: Perturbation integral (PI-km/s) as a function of the inclination of the orbits for different values of the semimajor axis.

Then, for all the other orbits, the integration made over one revolution is normalized by dividing its value by the period of the orbit and multiplying the result by the period of the reference orbit. In this way, the effect of increasing the period is removed from the results and comparisons can be made between orbits with different periods. In the calculations made in the present paper, the orbit with semimajor axis of $1670 \mathrm{~km}$ is chosen to be the reference orbit. Lines with different colors are used to show the results for several values of the semimajor axis of the orbit, thus permitting the presentation of a more general study where inclination and semimajor axis can be varied.

Figure 1 shows some details of the orbits. First of all, it is clear that they are symmetric with respect to the inclination of 90 degrees. This fact indicates that prograde and retrograde orbits have the same perturbation effects. This conclusion can be explained by physical considerations, because the forces used in the dynamical model (potential of Europa and thirdbody perturbation of Jupiter) do not depend on the sense of the orbits. It is also noted that when considering equatorial orbits, the perturbation integral first decreases with the semimajor axis (when it goes from $1670 \mathrm{~km}$ to $1716 \mathrm{~km}$ ) and then it increases with this parameter (after $1716 \mathrm{~km}$ ). This is due to the fact that when near Europa, the terms due to the gravity field of this moon are larger, when compared to the third-body perturbation, and those effects are more important when near the body; on the opposite side, when going far from the body, the third body dominates the perturbation and those effects increase when the distance from Europa increases. It means that Figure 1 shows not only the best value for the semimajor axis but also the cost of using different values, allowing mission designers to choose the best orbit for their mission. This point will be discussed in more detail later, when each component of the force is analyzed. 


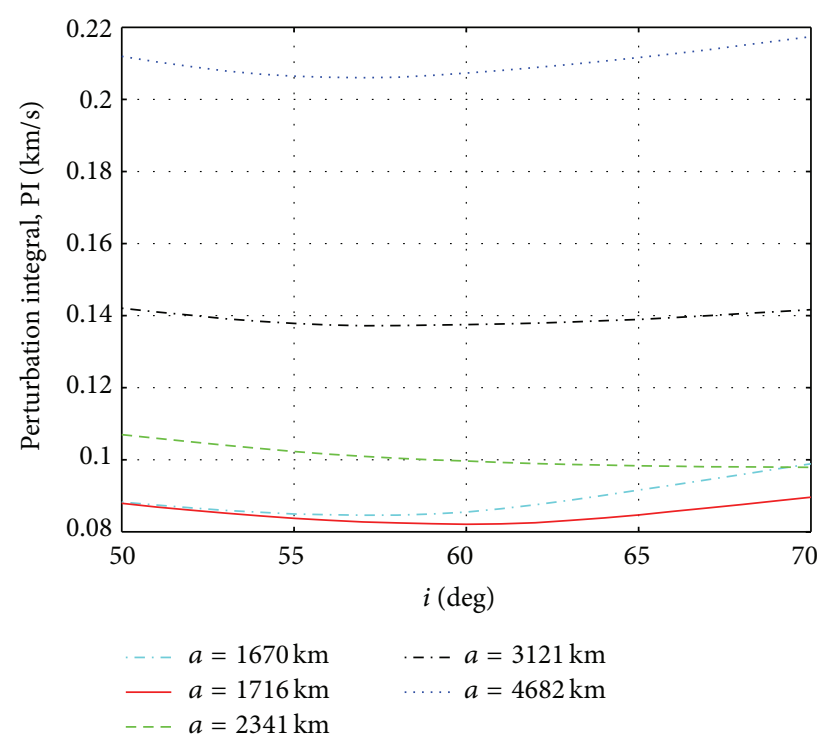

FIgURE 2: Perturbation integral (PI-km/s) as a function of the inclination of the orbits in the range 50-70 degrees.

Figure 2 shows the region of minimum perturbation in detail, by plotting the value of the perturbing integral for inclinations between 50 and 70 degrees in steps of 1 degree. For the orbit with semimajor axis of $1670 \mathrm{~km}$, the minimum is located at the inclination of 57 degrees. This minimum has the value of PI $=0.08461 \mathrm{~km} / \mathrm{s}$. This value can be determined with more accuracy by reducing the increment of the inclination, if desired. For orbits with semimajor axis of $1716 \mathrm{~km}$, the minimum occurs at the inclination of 60 degrees and the corresponding value is PI $=0.08209 \mathrm{~km} / \mathrm{s}$. Orbits with semimajor axis of $2341 \mathrm{~km}$ have minimum on the inclination of 72 degrees, with a corresponding value of PI $=$ $0.09779 \mathrm{~km} / \mathrm{s}$. This value is a little bit out of the plot shown in Figure 2. Considering orbits with semimajor axis of $3121 \mathrm{~km}$, the minimum occurs at the inclination of 57 degrees and its value is $\mathrm{PI}=0.13720 \mathrm{~km} / \mathrm{s}$. Finally, orbits with semimajor axis of $4682 \mathrm{~km}$ have minimum near the inclination of 57 degrees, showing a value of PI $=0.20601 \mathrm{~km} / \mathrm{s}$. Those minima represent orbits with more compensation of effects between the terms that come from the gravity field of Europa and the third-body perturbation from Jupiter. They represent the best values for the inclination, for each value of the semimajor axis, to place a satellite from the point of view of receiving less total perturbation. It is expected that those orbits will deviate less from a keplerian orbit in a given time and therefore require less fuel for station-keeping maneuvers.

To better understand the facts observed, it is useful to take a look in more detail at the integrals. Figures 3,4 , and 5 show the evolution of the magnitude of the integral for all the forces considered in the dynamical model, individually, as a function of the time for orbits with inclinations $0,50^{\circ}$, and $90^{\circ}$. The vertical axis shows the PI $(\mathrm{km} / \mathrm{s})$ and the horizontal axis shows the time (sec) for an orbit with semimajor axis of $1670 \mathrm{~km}, g=270^{\circ}, h=90^{\circ}$. A similar study was made for the values of semimajor axis of $1716 \mathrm{~km}, 2341 \mathrm{~km}, 3121 \mathrm{~km}$,

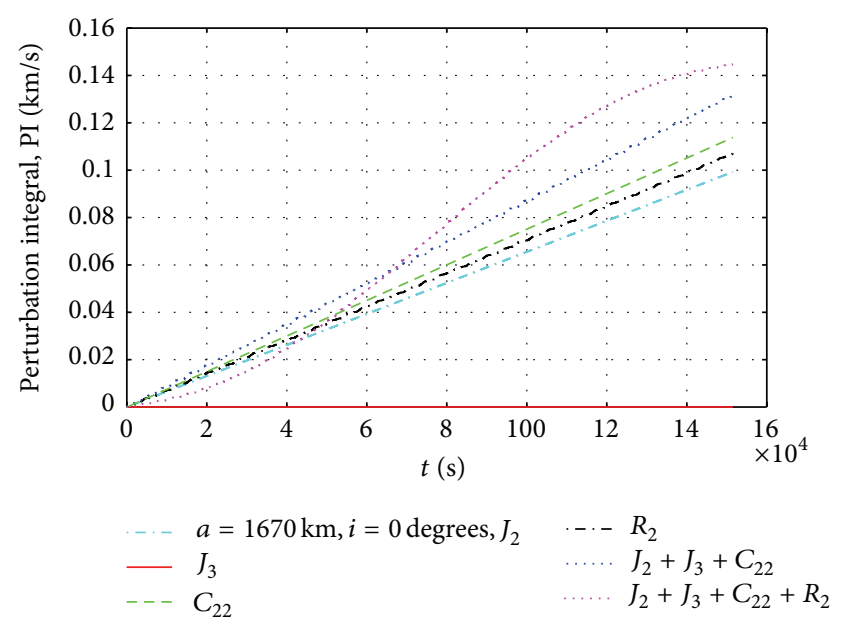

FIgURE 3: Perturbation integral (PI) with the magnitude of each force as a function of time for orbits with semimajor axis of $1670 \mathrm{~km}$ and inclination of zero degree; $g=270$ degrees and $h=90$ degrees.

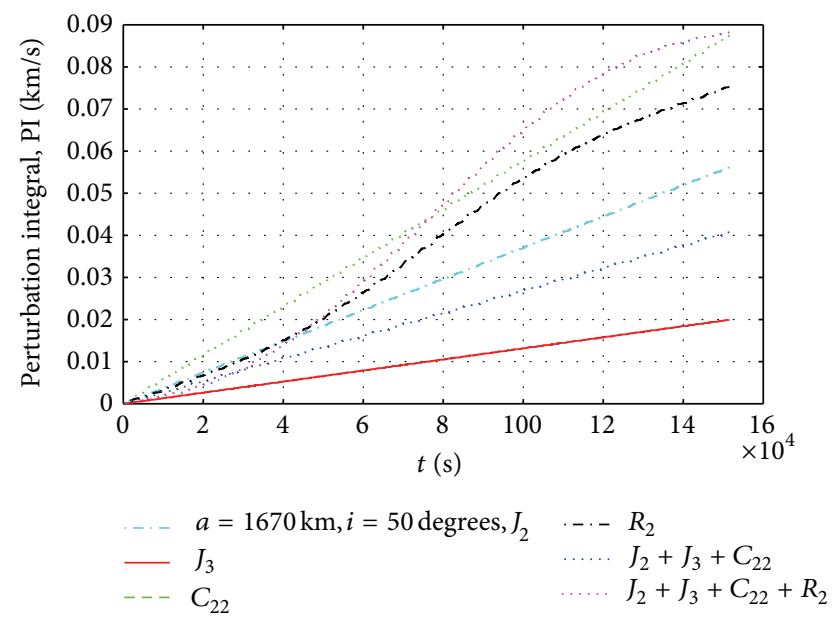

Figure 4: Perturbation integral (PI) with the magnitude of each force as a function of time for orbits with semimajor axis of $1670 \mathrm{~km}$ and inclination of 50 degrees; $g=270$ degrees and $h=90$ degrees.

and $4682 \mathrm{~km}$, always considering inclinations of 0 (equatorial orbits), $50^{\circ}$ (near-critical inclination orbits), and $90^{\circ}$ (polar orbits), and the results are very similar; therefore, they are not shown here. After that, Figure 6 is presented to condensate all the results obtained. It shows the PI in the vertical axis and the semimajor axis in the horizontal axis. This more detailed study can explain some of the characteristics observed in the previous figures.

The first fact that can be explained is the variation of the perturbing integral with respect to the semimajor axis. It was noticed that its value decreases from the orbit with semimajor axis of $1670 \mathrm{~km}$ to the orbit with semimajor axis of $1716 \mathrm{~km}$, but it is visible that there are two regions (around 40-50 degrees and 130-140 degrees) where the values are very near each other. Then the orbit with semimajor axis of $2341 \mathrm{~km}$ follows, which alternates higher and lower values of the PI with respect to the previous two orbits, 


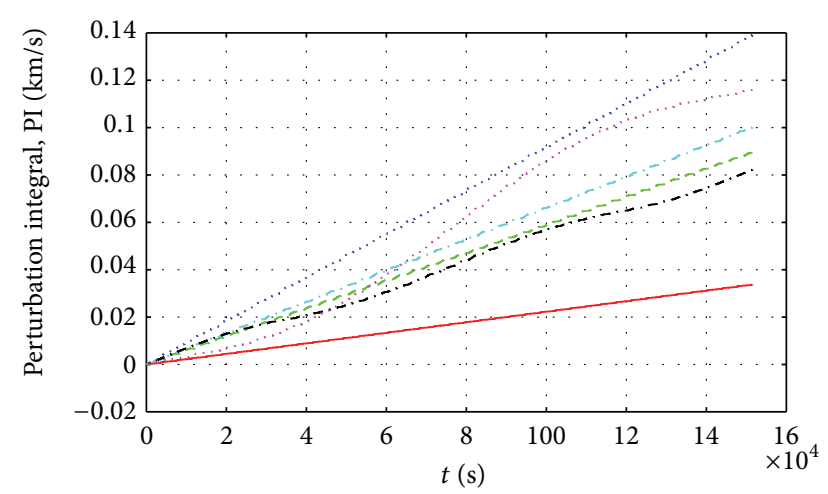

$$
\begin{array}{llll}
-\cdot a=1670 \mathrm{~km}, i=90 \text { degrees, } J_{2} & \cdots- & R_{2} \\
- & J_{3} & \ldots & J_{2}+J_{3}+C_{22} \\
--- & C_{22} & \ldots & J_{2}+J_{3}+C_{22}+R_{2}
\end{array}
$$

FIGURE 5: Perturbation integral (PI) with the magnitude of each force as a function of time for orbits with semimajor axis of $1670 \mathrm{~km}$ and inclination of 90 degrees; $g=270$ degrees and $h=90$ degrees.

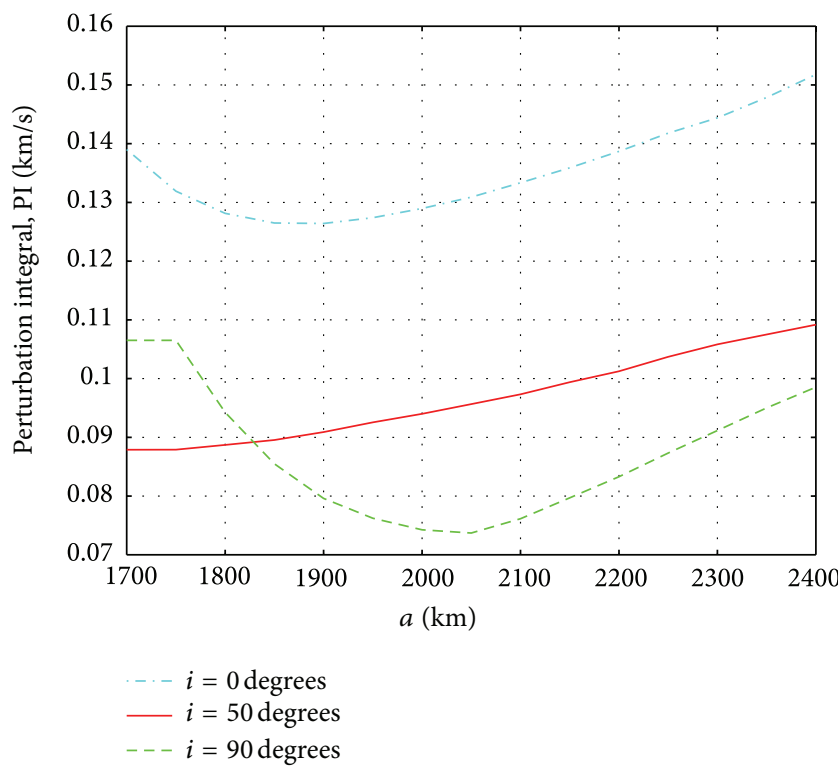

FIgURE 6: Perturbing integral (PI-km/s) as a function of the semimajor axis for orbits with inclinations of 0,50 , and 90 degrees.

depending on the inclination of the orbit. After that, the values are always increasing (Figure 1). Figures 3 to 5 explain this phenomenon. For values of semimajor axis closer to Europa, the perturbations due to the potential of Europa $\left(J_{2}, J_{3}\right.$, and $\left.C_{22}\right)$ are, in general, more important than the third-body perturbation of Jupiter, due to the high level of compensations among the different terms of the gravity potential. The exceptions occur for near-critical inclinations, where the total effects of the potential of Europa are smaller than the third-body perturbation due to Jupiter. Therefore, by increasing the distance of the orbit from Europa, the perturbations due to the $J_{2}+J_{3}+C_{22}$ terms have their effect decreased, thus reducing the total perturbation forces acting on the spacecraft. The increase of the less important (at this distance) third-body perturbation of Jupiter is not enough to compensate the decrease due to the potential of Europa. Therefore, the net result is a decrease in the value of the perturbation integral. But when this distance is getting greater, the opposite effect is noticed. The perturbations due to the potential of Europa become less important and the third-body perturbation of Jupiter dominates the motion of the spacecraft. After this point, the values of the PI will always increase, since the spacecraft is going closer to Jupiter and far from Europa. Therefore, the existence of a semimajor axis that represents a minimum value for the perturbing integral is expected that would be the ideal semimajor axis to place the spacecraft, with respect to this criterion. Figure 6 shows the results of the PI as a function of the semimajor axis. It is observed that the value of the semimajor axis that gives the minimum value is near $1900 \mathrm{~km}$ for the equatorial orbits, $1700 \mathrm{~km}$ for near-critical orbits, and $2050 \mathrm{~km}$ for polar orbits. This minimum is about $30 \%$ smaller than the maximum value for the polar case, $20 \%$ for the near-critical situation, and $17 \%$ for the equatorial orbits; therefore, an orbit substantially less perturbed can be obtained by choosing the best altitude, if no other constraint of the mission imposes something different. It means that there is a good potential of finding orbits that requires less station-keeping maneuvers for this semimajor axis. Detailed calculations omitted here show that, after a certain value of the semimajor axis, the third-body perturbation of Jupiter dominates so much the dynamics such that the potential of Europa can be neglected.

Studying the results in more detail, it is visible that the sum of the contributions of each individual effect is different from the total effect, as shown by Figures 3 to 5 . This is explained by the fact that the perturbations have positive and negative signs with respect to each other. Therefore, there are compensations which mean that, for a given position of the spacecraft, some perturbing forces are trying to make the orbit deviate from Keplerian, while some other forces are working in the opposite direction, thus helping the control system to maintain the orbit keplerian and then reducing the values of the integral and the fuel consumption. A clear indication of this fact is shown in Figure 5, where the perturbing integral related to the total forces is smaller than the perturbing integral of the effects of the terms only due to the potential of Europa. It clearly means that the third-body perturbation of Jupiter is helping to control the system and reducing the magnitude of the perturbing forces. This is an interesting characteristic of the approach used here to evaluate the effects of the perturbing forces and emphasize the importance of finding the most important perturbations involved in the dynamics.

The next interesting fact that can be explained is the variation of the perturbing integral with respect to the inclination of the orbit of the spacecraft. Figures 3 to 5 show a comparison between the polar, near-critical (50 degrees of inclination), and equatorial orbits with semimajor axis of $1670 \mathrm{~km}$. Looking at the individual effect of each perturbation, some information can be obtained. The contribution of $J_{3}$ goes from near zero in the equatorial orbits to around $0.02 \mathrm{~km} / \mathrm{s}$ for the near-critical orbits and then close to $0.38 \mathrm{~km} / \mathrm{s}$ in the polar case. Regarding $J_{2}$, its contribution 
goes from near $0.1 \mathrm{~km} / \mathrm{s}$ in the equatorial orbits to around $0.058 \mathrm{~km} / \mathrm{s}$ for the near-critical orbits and then close to $0.1 \mathrm{~km} / \mathrm{s}$ again for the polar orbits. For the $C_{22}$, its contribution goes from near $0.115 \mathrm{~km} / \mathrm{s}$ in the equatorial orbits to around $0.085 \mathrm{~km} / \mathrm{s}$ for the near-critical orbits and then close to $0.09 \mathrm{~km} / \mathrm{s}$ for the polar orbits. As a result of those effects, the total contribution of the potential of Europa goes from near $0.13 \mathrm{~km} / \mathrm{s}$ in the equatorial orbits to around $0.04 \mathrm{~km} / \mathrm{s}$ for the near-critical orbits and then close to $0.14 \mathrm{~km} / \mathrm{s}$ for the polar orbits. Those numbers show clearly that the potential plays an important role in the existence of the minimum with respect to the inclination. It is interesting to note that the contribution of the $C_{22}$ term dominates the scene for the equatorial and near-critical orbits and gets a close second place to the $J_{2}$ effects for polar orbits. It is also necessary to take into account the compensations by having different pointing in the direction of the forces. Equatorial and polar orbits have more aligned effects of the individual terms of the potential of Europa, while near-critical orbits have more compensations, therefore reducing the total contribution of the terms that comes from Europa. This is the main reason of the existence of the minimum values for the perturbation integral near the critical inclinations. It is also clear that, for equatorial and polar orbits, the effects of the total potential are larger than all the individual elements, while, for the near-critical orbits, the total effects of the potential are smaller than the effects of $J_{2}$ and $C_{22}$ individually. The net result is that the total contribution from the potential of Europa is about three times larger for equatorial and polar orbits, when compared to the near-critical orbits. Using the same comparison, the effect of the third-body perturbation from Jupiter decreases from the equatorial orbits, where it is near 0.11 , to the nearcritical orbits, where it is near 0.075 . Then, it increases by a small amount when going to polar orbits, reaching a value near 0.08 . In terms of total value, there is an increase of about $30 \%$ for the perturbation effects of polar orbits when compared to the near-critical orbits. It should also be noted that, for the polar orbits, the value of the integral for the total perturbations is smaller than the value obtained when considering only the potential of Europa, so the potential of Europa is responsible for the minimum. Regarding a comparison between equatorial and polar orbits, the results show that the main reason for the higher costs for the equatorial orbits lies in the alignment of the third-body and the potential perturbations. For the equatorial and near-critical orbits, the value for the integral of the total perturbations is larger than the value for the potential, while, for the polar orbits, this total value is smaller than the value due to the potential alone, meaning that there are compensations among the terms. In other words, the third-body perturbation helps to control the system for polar orbits, and this fact makes the costs smaller than the equivalent ones for equatorial orbits.

A similar study for orbits with semimajor axis equal to $1716 \mathrm{~km}$ shows similar results. The main difference is that the magnitude of all effects due to the potential of Europa decreases, while the effects of the third-body perturbation of Jupiter are increased, as a result of the longer distance from Jupiter. When considering orbits with semimajor axis of $2341 \mathrm{~km}$, the results show that the third-body perturbation of Jupiter starts to be the main force in the dynamics of the spacecraft. The potential of Europa plays the role of working against the third-body perturbation, therefore reducing the value of the integral. But these compensating effects are not the same for all the orbits studied. It is almost zero for equatorial orbits, small for near-critical orbits, and large for polar orbits. This fact generates an interesting result. The larger effects in the polar orbits make the value of the perturbing integral decrease and the minimum of the graph is no longer sharp. Figure 1 shows that there is a sharp local maximum at the polar orbits. Therefore, this is one more fact to be considered when choosing an orbit for a spacecraft around Europa. For orbits with semimajor axis of $3121 \mathrm{~km}$, the simulations show that all the elements of the potential of Europa increase its participation in polar orbits. The gap between the effects of the third-body perturbation from Jupiter and the total effects increases, but the net result is an increase in the total value. From this point, in terms of the semimajor axis of the orbits, the effects of the potential of Europa are negligible and the problem becomes a pure thirdbody perturbation. Simulations for orbits with semimajor axis of $4682 \mathrm{~km}$ confirm this result.

3.3. Studying Frozen Orbits. An important application of the present research is in the search for frozen orbits, which are orbits where the natural variations of the orbital elements are minimized by a proper choice of the initial values of the orbital parameters. Those orbits are more stable and they require less fuel consumption for station-keeping maneuvers, therefore extending the lifetime of the mission. The results shown in the present paper give a number (the PI) that can represent the total effects that the perturbing forces make in a spacecraft for a specific orbit, so it is possible to find which are the values for the semimajor axis that receives fewer perturbations compared to the others. Therefore, it is a good idea to search for frozen orbits in which semimajor axes are near the ones where the perturbations are minimized. Based on that analysis, it is expected that the best value for the semimajor axis of the frozen orbit is $2341 \mathrm{~km}$, since we are using an inclination of 90 degrees for all the frozen orbits and this is the value of semimajor axis that generates the less perturbed orbit, as seen in Figure 1. Using the same arguments, it is expected that the second best value for the semimajor axis is $1716 \mathrm{~km}$, followed by $1670 \mathrm{~km}, 3121 \mathrm{~km}$, and finally $4682 \mathrm{~km}$. Figures 7 to 11 were obtained by using (5), (10), and (11), which are presented by Carvalho et al. [14]. They take into account the gravitational attraction of a third body and the nonuniform distribution of the mass of Europa $\left(J_{2}\right.$ and $\left.J_{3}\right)$. These equations were plugged in Lagrange's planetary equations and numerically integrated using the software Maple. Figures 7 to 11 show the frozen orbits. The integration time of all the orbits is 500 days. It is clear that the results are well inside the expected ones from the analysis of integrals of the forces. Figure 7 shows the orbits with the semimajor axis of $2341 \mathrm{~km}$, for eccentricities of $0.001,0.002,0.003,0.004$, and 0.005 . The maximum amplitudes of variations are around 110 degrees in the argument of periapsis and 0.01 in eccentricity, in the worst case, but a situation with almost constant values exists, for eccentricities of 0.005 . Figure 8 shows similar 


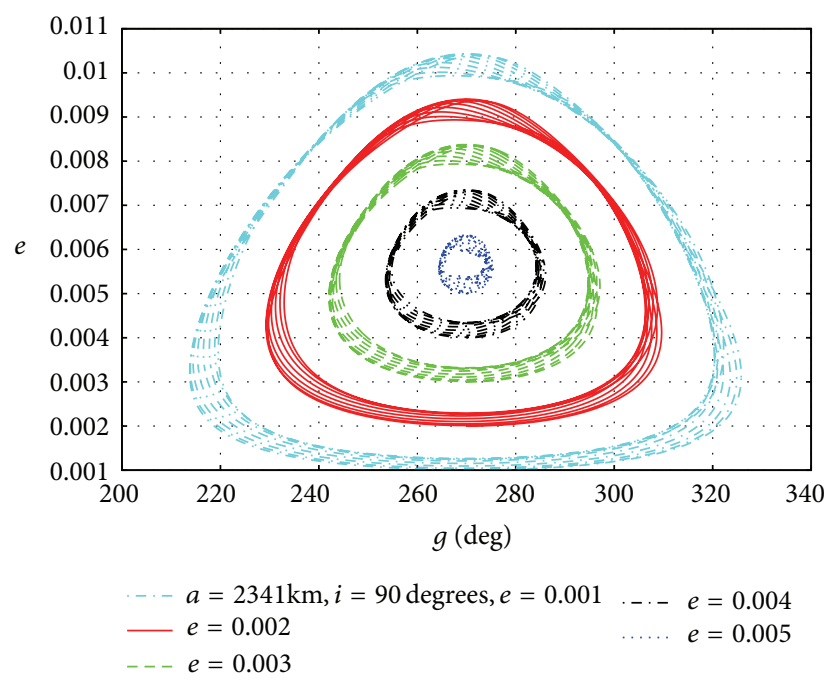

FIgURE 7: Orbits with semimajor axis of $2341 \mathrm{~km}$ and eccentricities of $0.001,0.002,0.003,0.004$, and 0.005 .

orbits but now with the semimajor axis of $1716 \mathrm{~km}$, for the same eccentricities used in the previous case. The maximum amplitude now for the variations is around 150 degrees for the argument of periapsis and 0.059 in eccentricity, in the worst case, and around 100 degrees in the argument of periapsis and 0.05 in eccentricity, in the best case, which indicates that the first orbits are much better, in terms of having smaller amplitudes of variation. Figure 9 shows the same orbits using now the semimajor axis of $1670 \mathrm{~km}$, the next best value, for the same eccentricities used before. The results are similar to the previous case, but with amplitudes a little bit larger for all the values of the eccentricity used. Figures 10 and 11 show the same orbits for the situations where the PI is larger than the previous cases, with semimajor axis of $3132 \mathrm{~km}$ and $4682 \mathrm{~km}$, respectively. The orbits are now not limited in the argument of periapsis, due to the larger perturbations. Of course there are many other aspects to be considered when planning a mission, like other values for the eccentricities of the orbits, goals and constraints of the mission, and so forth, but the results presented here show that the integral of the perturbing forces can be an interesting tool for mission designers during the orbit selection process for a real mission, constituting one more element to be analyzed.

\section{Conclusions}

This paper studied the total perturbation acting on a spacecraft in circular orbits around Europa, using an index based on the integral of the perturbing forces over the time. This definition proved to be useful in the evaluation of the perturbation forces acting on a spacecraft around Europa, therefore allowing good choices for the orbits. A study was then conducted to calculate the effects of the main terms of the potential of Europa $\left(J_{2}, J_{3}\right.$, and $\left.C_{22}\right)$ and the third-body perturbation due to Jupiter. The results were used to map orbits with respect to the perturbations acting on a spacecraft in each of these orbits. The results show several interesting

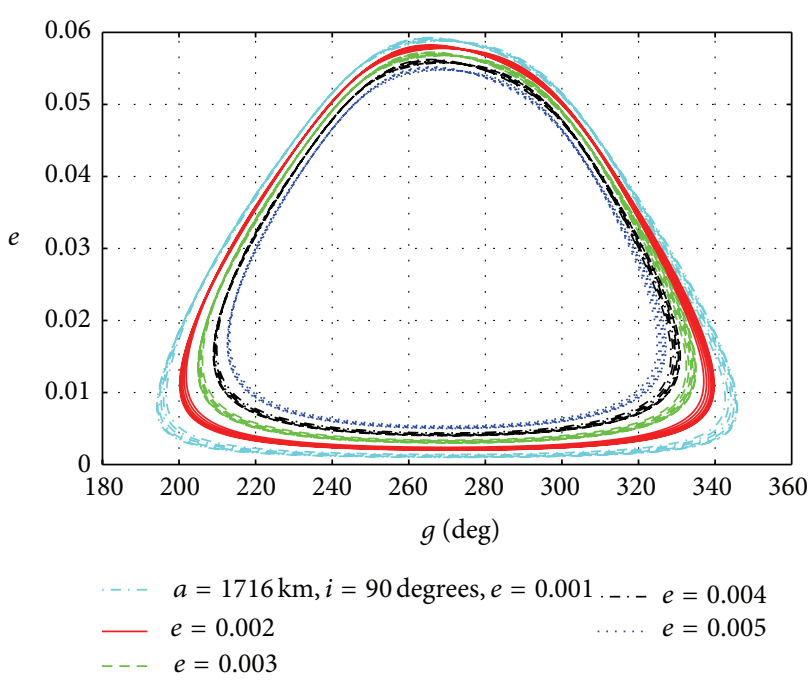

FIGURE 8: Orbits with semimajor axis of $1716 \mathrm{~km}$ and eccentricities of $0.001,0.002,0.003,0.004$, and 0.005 .

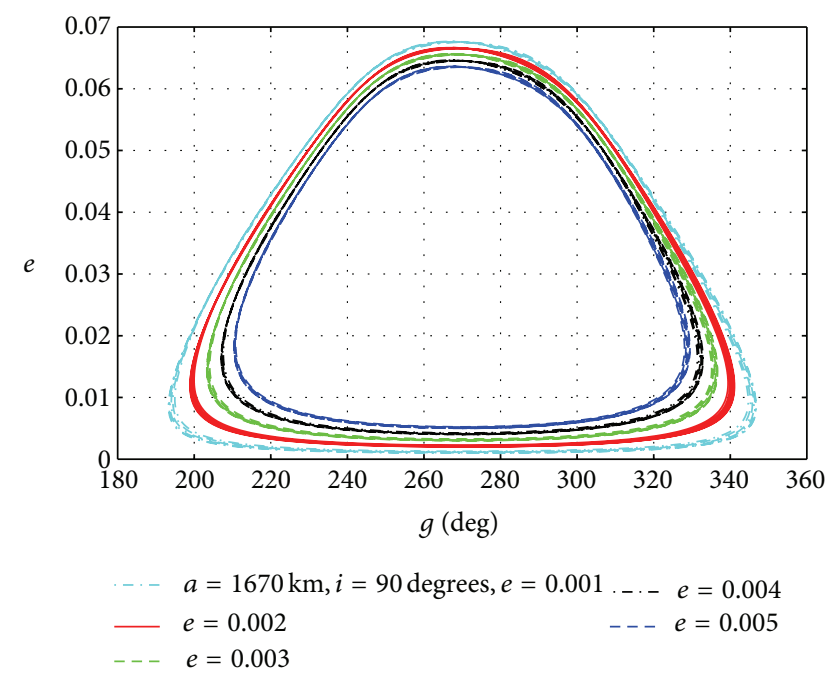

FIgURE 9: Orbits with semimajor axis of $1670 \mathrm{~km}$ and eccentricities of $0.001,0.002,0.003,0.004$, and 0.005 .

characteristics: (1) the existence of orbits with minimum values for this index with respect to the semimajor axis, for a fixed inclination; (2) the existence of orbits with minimum values for this index with respect to the inclination, for a fixed semimajor axis; (3) the role of each individual term of the perturbing forces; (4) the forces having different directions with respect to each other, so, for a given position of the spacecraft, some forces are acting to destroy the keplerian orbit, while others are working together with the propulsion system to keep the keplerian orbit, therefore reducing the fuel consumption; (5) orbits with semimajor axis of $2341 \mathrm{~km}$ having a flat minimum, with values for the integrals about the same for inclinations ranging from 50 to 90 degrees; (6) the perturbation forces acting on the spacecraft being symmetric with respect to the inclination; therefore, prograde and retrograde orbits have the same costs; 


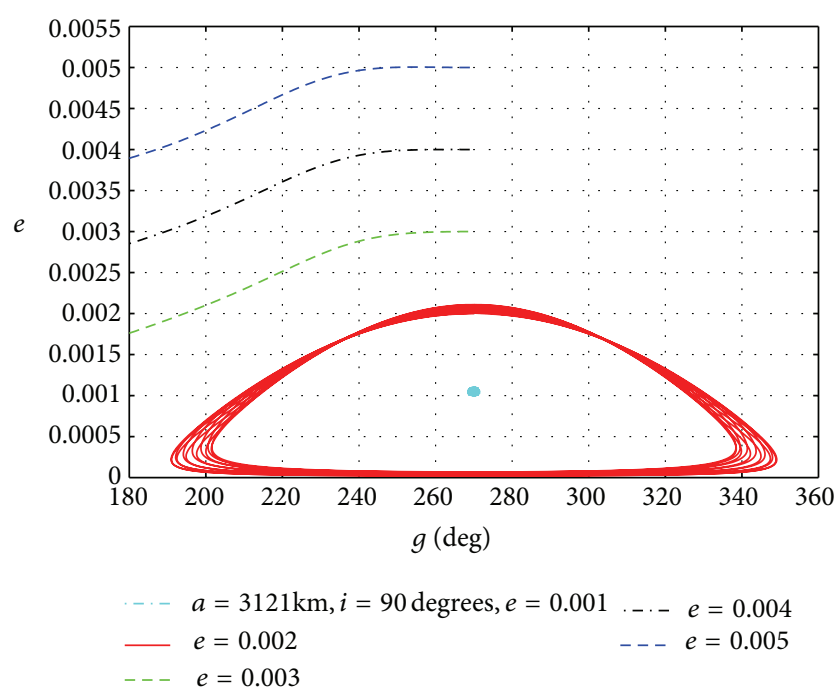

FIGURE 10: Orbits with semimajor axis of $3121 \mathrm{~km}$ and eccentricities of $0.001,0.002,0.003,0.004$, and 0.005 .

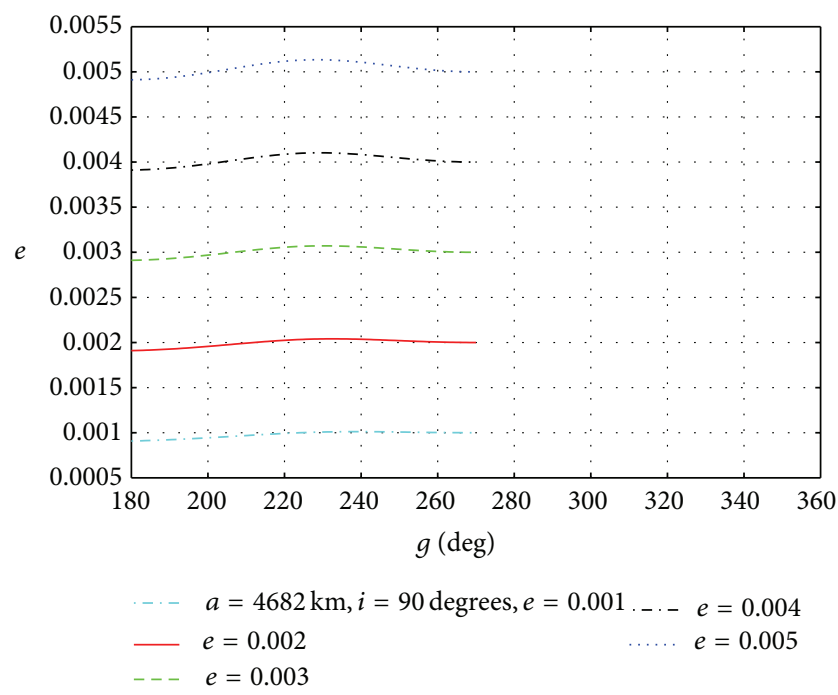

FIGURE 11: Orbits with semimajor axis of $4682 \mathrm{~km}$ and eccentricities of $0.001,0.002,0.003,0.004$, and 0.005 .

(7) in all the situations studied here, the equatorial orbits having perturbation integrals with higher values than the other orbits. After that, the present study is completed by using this index to choose the semimajor axis of potential frozen orbits. The results showed that the amplitudes of the variations of the eccentricity and argument of periapsis are related to this index, with the minimum values generating more stable orbits. In this sense, the ideas developed here can be useful in the analysis of future missions, during the orbit selection process.

\section{Conflict of Interests}

The authors declare that there is no conflict of interests regarding the publication of this paper and that they do not have a direct financial relation that might lead to one.

\section{Acknowledgments}

The authors wish to express their appreciation for the support provided by Grant nos. 303070/2011-0, 473387/2012-3, and 304700/2009-6, from the National Council for Scientific and Technological Development (CNPq), and Grant nos. 2011/ 05671-5, 2011/09310-7, 2011/08171-3, and 2012/21023-6, from São Paulo Research Foundation (FAPESP), and the financial support from the National Council for the Improvement of Higher Education (CAPES).

\section{References}

[1] D. J. Scheeres, M. D. Guman, and B. F. Villac, "Stability analysis of planetary satellite orbiters: application to the Europa orbiter," Journal of Guidance, Control, and Dynamics, vol. 24, no. 4, pp. 778-787, 2001.

[2] M. Lara and S. Ferrer, "Secular motion around tri axial, synchronously orbiting, planetary satellites: application to Europa," Chaos: An Interdisciplinary Journal of Nonlinear Science, vol. 15, no. 4, Article ID 043101, pp. 1-13, 2005.

[3] M. Lara and J. F. San-Juan, "Dynamic behavior of an orbiter around Europa," Journal of Guidance, Control, and Dynamics, vol. 28, no. 2, pp. 291-297, 2005.

[4] M. Lara and R. Russell, "On the design of a science orbit about Europa," in Proceedings of the 2006 AAS/AIAA Space Flight Mechanics Meeting, Tampa, Fla, USA, January 2006.

[5] M. E. Paskowitz and D. J. Scheeres, "Design of science orbits about planetary satellites: application to Europa," Journal of Guidance, Control, and Dynamics, vol. 29, no. 5, pp. 1147-1158, 2006.

[6] M. Lara and R. P. Russell, "Computation of a science orbit about Europa," Journal of Guidance, Control, and Dynamics, vol. 30, no. 1, pp. 259-263, 2007.

[7] M. Lara, "Simplified equations for computing science orbits around planetary satellites," Journal of Guidance, Control, and Dynamics, vol. 31, no. 1, pp. 172-181, 2008.

[8] M. P. Possner and D. J. Scheeres, "Control of science orbits about planetary satellites," Journal of Guidance, Control, and Dynamics, vol. 32, no. 1, pp. 223-231, 2009.

[9] R. P. Russell and A. T. Brinckerhoff, "Circulating eccentric orbits around planetary moons," Journal of Guidance, Control, and Dynamics, vol. 32, no. 2, pp. 423-435, 2009.

[10] M. Lara, "Three-body dynamics around the smaller primary. Application to the design of science orbits," Journal of Aerospace Engineering, Sciences and Applications, vol. 2, no. 1, pp. 53-65, 2010.

[11] J. P. S. Carvalho, A. Elipe, R. Vilhena de Moraes, and A. F. B. A. Prado, "Low-altitude, near-polar and near-circular orbits around Europa," Advances in Space Research, vol. 49, no. 5, pp. 994-1006, 2012.

[12] J. P. S. Carvalho, D. C. Mourão, A. Elipe, R. Vilhena de Moraes, and A. F. B. A. Prado, "Frozen orbits around Europa," International Journal of Bifurcation and Chaos in Applied Sciences and Engineering, vol. 22, no. 10, Article ID 1250240, 13 pages, 2012. 
[13] J. E. Chambers, "A hybrid symplectic integrator that permits close encounters between massive bodies," Monthly Notices of the Royal Astronomical Society, vol. 304, no. 4, pp. 793-799, 1999.

[14] J. P. S. Carvalho, R. Vilhena de Moraes, and A. F. B. A. Prado, "Dynamics of artificial satellites around Europa," Mathematical Problems in Engineering, vol. 2013, Article ID 182079, 7 pages, 2013.

[15] A. F. B. A. Prado, "Searching for orbits with the minimum fuel consumption for station-keeping maneuvers: application to luni-solar perturbations," Mathematical Problems in Engineering, vol. 2013, Article ID 415015, 11 pages, 2013.

[16] C. R. H. Solórzano and A. F. B. A. Prado, "Third-body perturbation using a single averaged model: application in nonsingular variables," Mathematical Problems in Engineering, vol. 2007, Article ID 40475, 14 pages, 2007.

[17] R. A. Broucke, "Long-term third-body effects via double averaging," Journal of Guidance, Control, and Dynamics, vol. 26, no. 1, pp. 27-32, 2003.

[18] A. F. B. A. Prado, "Third-body perturbation in orbits around natural satellites," Journal of Guidance, Control and Dynamics, vol. 26, no. 1, pp. 33-40, 2003.

[19] R. C. Domingos, R. V. Moraes, and A. F. B. A. Prado, "Thirdbody perturbation in the case of elliptic orbits for the disturbing body," Mathematical Problems in Engineering, vol. 2008, Article ID 763654, 14 pages, 2008.

[20] X. Liu, H. Baoyin, and X. Ma, "Long-term perturbations due to a disturbing body in elliptic inclined orbit," Astrophysics and Space Science, vol. 339, no. 2, pp. 295-304, 2012.

[21] S. W. McCuskey, Introduction to Celestial Mechanics, AddisonWesley Publishing Company, Reading, Mass, USA, 1963.

[22] G. E. O. Giacaglia, J. P. Murphy, and T. L. Felsentreger, "A semianalytic theory for the motion of a lunar satellite," Celestial Mechanics, vol. 3, no. 1, pp. 3-66, 1970. 


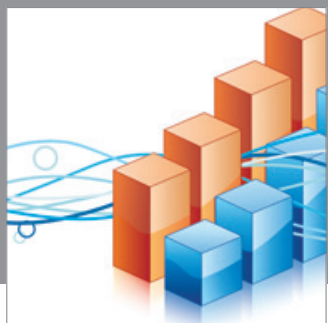

Advances in

Operations Research

mansans

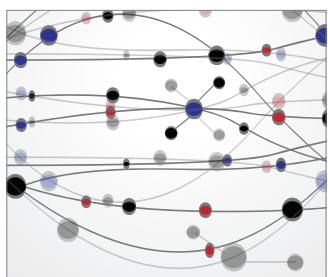

The Scientific World Journal
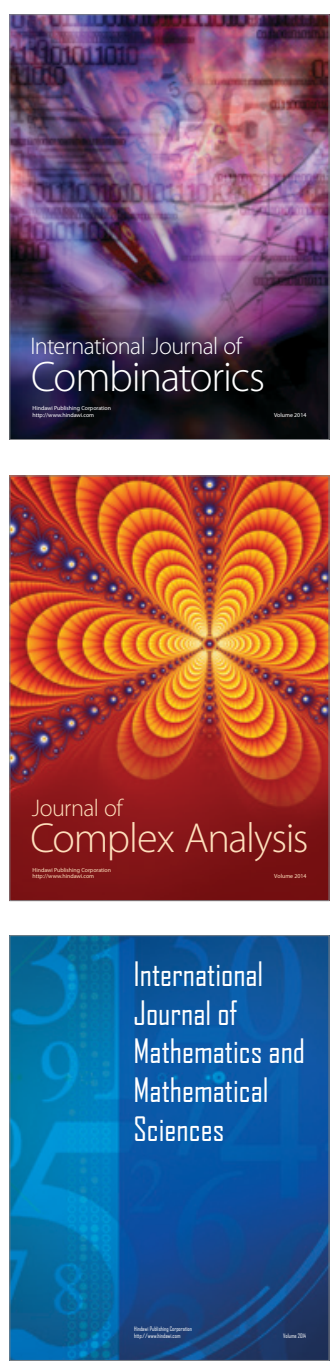
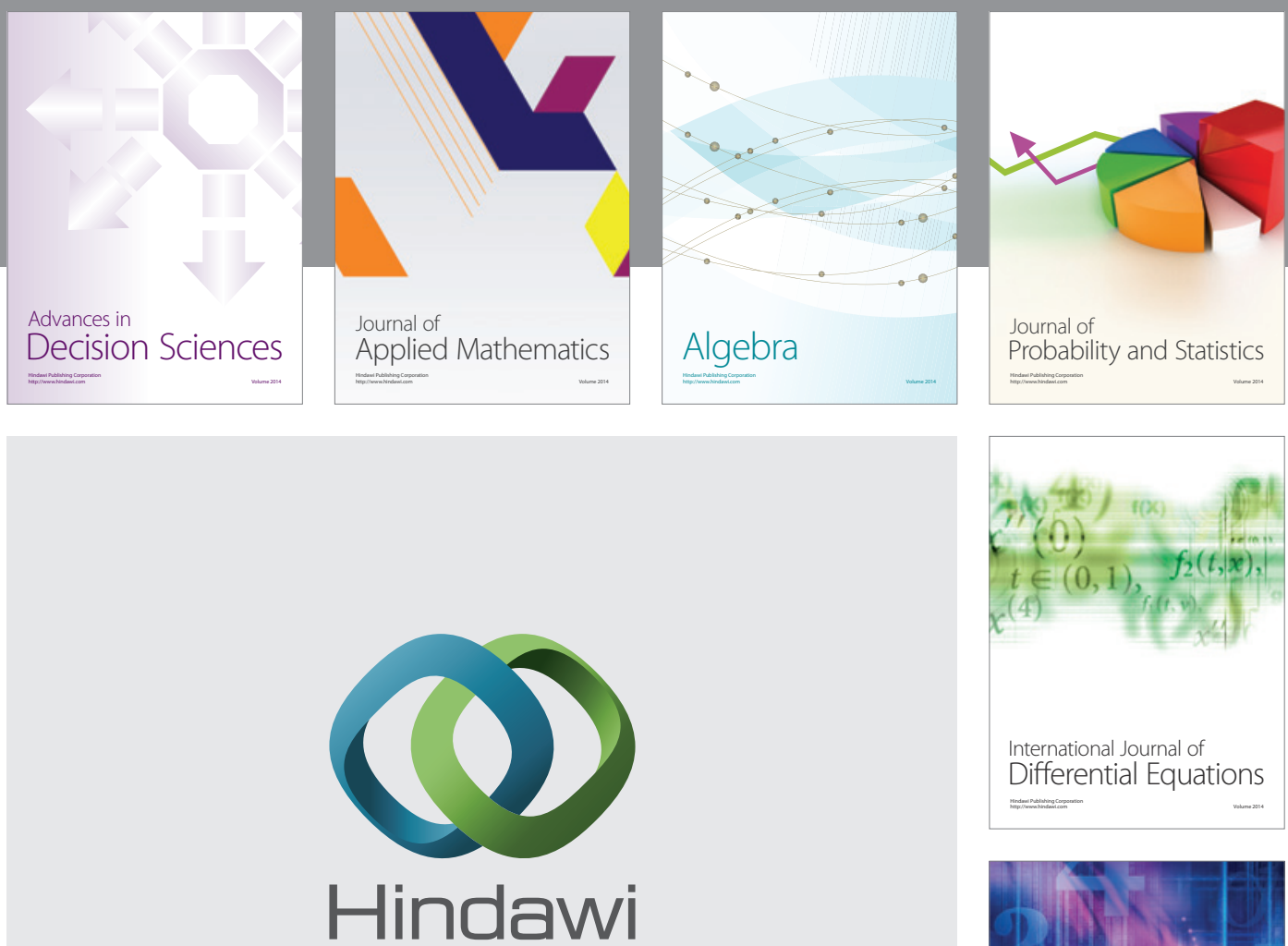

Submit your manuscripts at http://www.hindawi.com
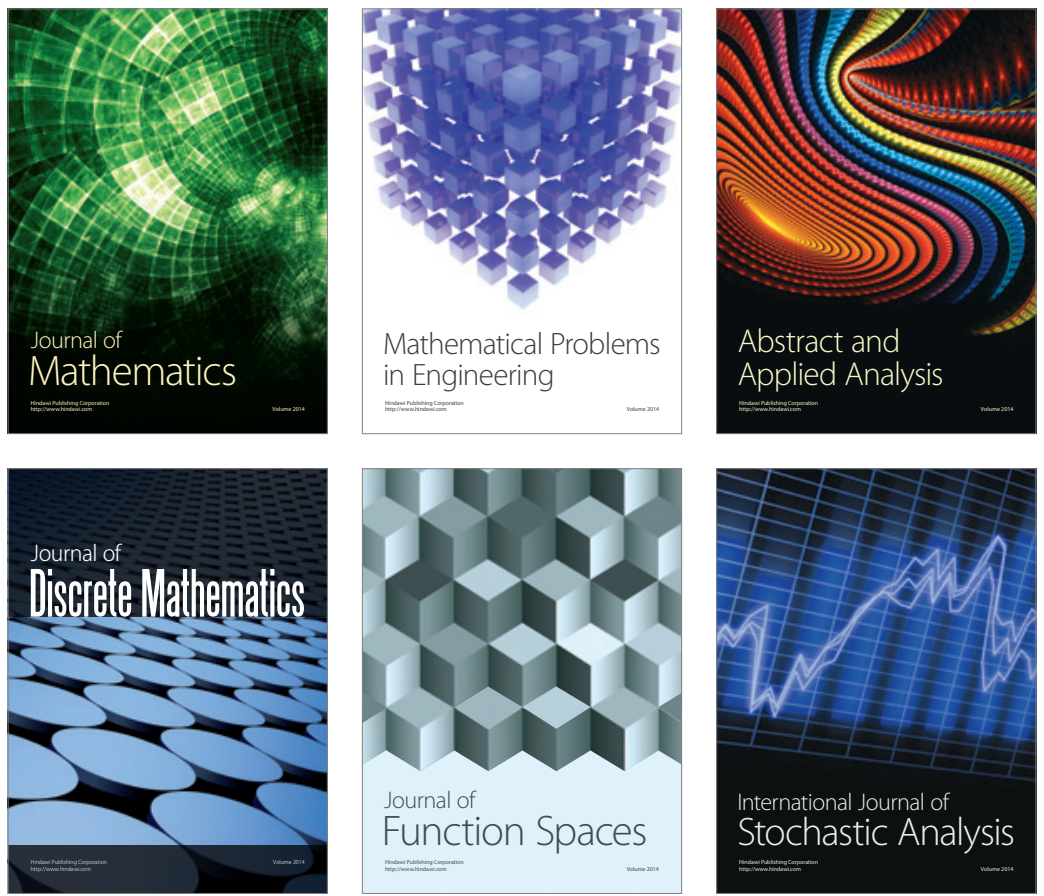

Journal of

Function Spaces

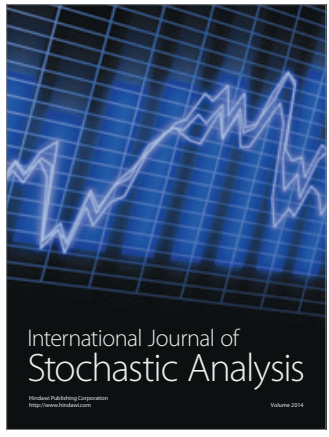

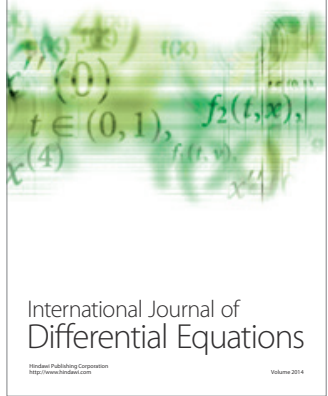
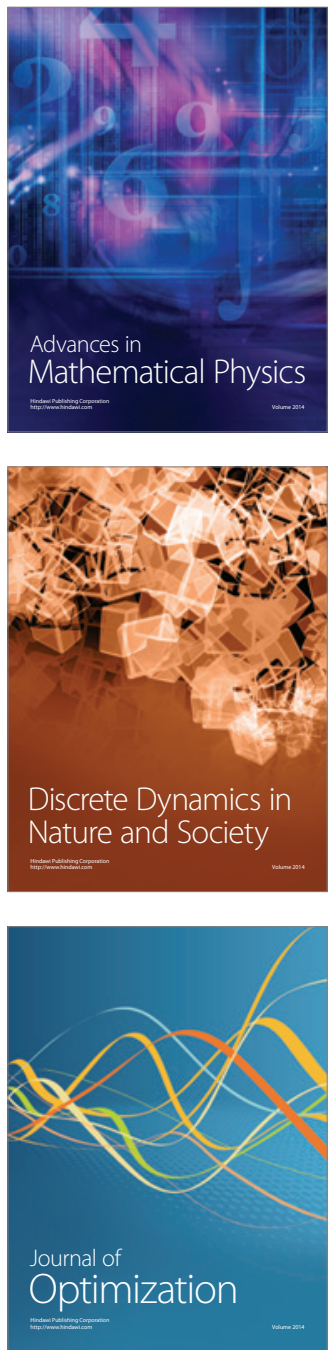